\title{
PLANEJAMENTO DAS ROTAS DE COLETA DE RESÍDUOS SÓLIDOS DOMICILIARES ESPECIAIS POR MEIO DE UM SISTEMA DE INFORMAÇÕES GEOGRÁFICAS PARA TRANSPORTE: APLICAÇÃO DO ALGORITMO DO PROBLEMA DE ROTEIRIZAÇÃO DOS VEÍCULOS COM JANELA DE TEMPO
}

\author{
Gustavo Andrés Araque González \\ Pontifícia Universidade Católica do Rio de Janeiro-Puc-Rio \\ ing.gustavo.araque@hotmail.com \\ José Eugenio Leal \\ Pontifícia Universidade Católica do Rio de Janeiro-Puc-Rio \\ jel@puc-rio.br
}

\begin{abstract}
Resumo
O crescimento mundial da população e o processo de urbanização tem feito aumentar a produção nas grandes indústrias e o consumo de bens no mercado, aumentando a geração da quantidade de lixo das cidades nos últimos anos. Devido ao fenômeno anterior, o número de pontos para a coleta dos Resíduos Sólidos Domiciliares aumenta, gerando custos e distâncias maiores, impactando no transito urbano e diminuindo a produtividade e qualidade dos sistemas logísticos e de transporte para as empresas de serviço de limpeza. Como solução e apoio nos processos de roteirização para a coleta dos rejeitos, utilizou-se o SIG-T software TransCAD versão 5.0. com a aplicação da rotina do Problema de Roteirização de Veículos com Janela de Tempo (PRVJT) para o planejamento, execução e análise dos roteiros dos Caminhões no Programa de Remoção Gratuita da Companhia de limpeza Urbana COMLURB na zona sul da cidade do Rio de Janeiro.

Palavras-Chaves: Resíduos Sólidos Domiciliares; Programa de Remoção Gratuita; Sistemas de Informações Geográficas para Transporte; Problema de Roteirização dos Veículos com Janela de Tempo.
\end{abstract}

\begin{abstract}
The global population growth and the urbanization process has made increasing production in major industries and consumer goods in the market, increasing the generation amount of garbage from cities in recent years. Because of the previous phenomenon, the number of points for the collection of Household Solid Waste increases, generating costs and longer distances, impacting the urban traffic and decreasing the productivity and quality of logistics and transport systems for cleaning service companies. As a solution and support in routing processes for the collection of waste, we used the GIS-T TransCAD software version 5.0. with the application of Vehicle Routing Problem with Time Window (VRPTW) routine for the planning, execution and analysis of routes of trucks in Cleaning Company's Free Removal Program Urban -COMLURB in the south of Rio de Janeiro city.

Keywords: $\quad$ Household Solid Waste; Free Removal Program; Geographic Information System for Transportation; Vehicle Routing Problem with Time Windows.
\end{abstract}

\section{INTRODUÇÃO: RELEVÂNCIA E MOTIVAÇÃO}

O crescimento mundial da população e o processo de urbanização tem feito aumentar a produção nas grandes indústrias e o consumo de bens no mercado, aumentando a geração da quantidade dos Resíduos Sólidos das cidades nos últimos anos. Enquanto as empresas ganham um crescimento e benefício com este comportamento consumista, o modelo deixa 
problemas ambientais, sociais e de saúde pública no mundo inteiro.

De acordo com [10] "a geração de Resíduos Sólidos no Brasil cresceu desde o ano de 2008 (169658 ton./dia) até o ano 2013 (209.280 ton./dia), apresentando um acréscimo de 18,93\%". Com base nesses dados, os rejeitos gerados pela sociedade merecem um trato especial das gestões municipais com a implementação de estratégias a curto, médio e longo prazo que permitam controlar e gerenciar os sistemas de gestão de serviços de transporte, coleta e disposição final dos resíduos.

Na cidade do Rio de Janeiro "são gerados diariamente 10.000 toneladas de resíduos sólidos, sendo 49,42\% de lixo domiciliar, $32,47 \%$ de lixo público e o restante $(18,11 \%)$ compreendido pelas parcelas de grandes geradores (incluindo resíduos da construção civil), atendimento a emergência, resíduos dos serviços municipais de saúde e remoção gratuita". [3]

Como medida de atendimento de limpeza da cidade, a gestão dos Resíduos Sólidos é executada de maneira constante pelo governo do estado em conjunto com a Secretaria do Estado do Ambiente da cidade (SEA) e os serviços operacionais de limpeza da Companhia Municipal de Limpeza Urbana-COMLURB. Para o desenvolvimento dos requerimentos ambientais da cidade, são planejados e executados os programas especiais de atendimento ao cidadão. Um deles é o Programa de Remoção Gratuita (PRG) da COMLURB.

Uma das atividades principais no PRG é o processo de coleta, transporte e disposição final dos resíduos. Para o planejamento dos roteiros a serem percorridos pelos veículos na gestão dos rejeitos da cidade, são utilizadas técnicas empíricas baseadas na experiência do recurso humano, diminuindo a produtividade e qualidade do serviço no atendimento dos clientes.

Como solução e apoio nos processos de roteirização para a coleta de Resíduos Sólidos Domiciliares, existem técnicas de criação, processamento e análise de dados que aumentam a eficiência na tomada de decisões. Elas são conhecidas como os Sistemas de Informações Geográficas para o Transporte (SIG-T).

Portanto, no presente trabalho, a ênfase será dada ao problema de roteirização dos veículos na coleta de Resíduos Sólidos Domiciliares dentro do PRG, incluindo a coleta, transporte e disposição final dos rejeitos. Será utilizado o SIG-T como processo de planejamento logístico na coleta de resíduos sólidos na região sul da cidade no Rio de Janeiro, com o desenvolvimento do Problema de Roteirização dos Veículos com Janela de Tempo (PRVJT) para o planejamento, execução, simulação e análise do sistema de roteirização.

\section{RESÍDUOS SÓLIDOS DOMICILIARES}

Os Resíduos Sólidos Domiciliares (RSD) são conhecidos como os rejeitos gerados nas residências da sociedade. Pela sua composição, eles podem ser orgânicos ou inorgânicos.

Existe um conjunto de elementos interligados que permitem executar de forma eficiente o serviço de limpeza das prefeituras. São eles: geração, acondicionamento, coleta, estação de transferência e disposição final dos RSD. [20].

Definida como a fonte de origem dos rejeitos, a geração dos resíduos sólidos é o resultado das atividades diárias dos seres humanos, sendo influenciada por vários fatores: "densidade populacional, grau de desenvolvimento econômico do local, hábitos de consumo, variações sazonais e condições socioeconômicas das populações". [2]. O principal indicador de geração de resíduos sólidos nas cidades é o índice de geração per capita de resíduos. No Brasil, o índice apresenta um crescimento de geração per capita de 2008 até 2012 com valores de $1,080 \mathrm{Kg} / \mathrm{hab} . /$ dia até $1,2282 \mathrm{Kg} / \mathrm{hab}$./dia respectivamente.

Já o acondicionamento dos Resíduos Sólidos é a atividade de disposição interna dos rejeitos em recipientes sanitariamente adequados para a coleta de acordo com o tipo e a quantidade de resíduo gerada.

Em relação à coleta de Resíduos Sólidos Domiciliares, a mesma é definida como a "remoção dos resíduos sólidos para um local de armazenamento, processamento ou 
destinação final". [2]. Existem três modalidades principais: a primeira é a coleta porta a porta, caracterizada por coletar o rejeito em frente à residência do solicitante; a segunda é o posto de entrega voluntária, onde o morador se desloca até um local fixo de disposição de rejeitos para depositar os RSD; a última modalidade ocorre por meio de catadores, pessoas independentes que recolhem os Resíduos Sólidos Domiciliares inorgânicos e recicláveis.

As estações de transferência ou pontos de transbordo, são lugares de destinação temporária dos resíduos sólidos, para em seguida serem transportados até o destino final.

O último passo no gerenciamento integrado dos Resíduos Sólidos é a disposição final. De acordo com [15], existem três formas de disposição final dos RSD: o lixão caracteriza-se pela simples descarga de resíduos sobre o solo, sem medidas de proteção ao ambiente ou a saúde pública; a segunda é o aterro controlado, onde a disposição final dos rejeitos urbanos é feita no solo diminuindo o impacto ambiental; a última é o aterro sanitário, técnica mais favorável para o meio ambiente, onde os resíduos são dispostos em camadas de terra utilizando-se técnicas de engenharia para diminuir a área e volume dos mesmos.

\section{PROGRAMA DE REMOÇÃO GRATUITA DA COMLURB}

O Programa de Remoção Gratuita (PRG) é o serviço complementar de limpeza dos rejeitos que não pertencem ao grupo de coleta seletiva (inorgânicos) e ao grupo de coleta domiciliar (resíduos orgânicos). É conhecido como um subprograma da Diretoria de Coleta Seletiva da COMLURB, empresa responsável pelo serviço de limpeza da cidade do Rio de Janeiro. O objetivo do programa é atender aos cidadãos que estão fazendo pequenas reformas em seus domicílios, com o serviço das "solicitações de remoção de material de pequenas obras, de entulhos (restos de obra), objetos (telhas, tijolos, galhos, troncos e outros) e bens inservíveis (fogão, geladeira, banheira e outros)" [1].

O serviço pode ser solicitado de duas formas: serviço de tele-atendimento ao usuário por meio da linha 1746 ou pela internet. Para o sucesso da gestão de limpeza do programa, é necessária e fundamental a conexão e interação dos diferentes departamentos do programa.

O procedimento de remoção gratuita funciona da seguinte forma: o centro de atendimento recebe as ligações dos cidadãos por meio da linha gratuita de atendimento 1746; depois, essas ligações são convertidas em pedidos, fazendo um cadastro on-line com os dados necessários para prestar o serviço ao cliente $\left(\mathrm{N}^{\circ}\right.$ do pedido, data, hora, nome completo do cidadão, telefone, localização do logradouro, bairro, descrição da coleta, endereço, etc.); estas informações são armazenadas no Sistema Ambiental de Coleta - SAC que envia os requerimentos de pedido à gerência, a qual avalia e aprova cada um dos pedidos. Uma vez aprovados, entram no Programa de Operação Ambiental de Coleta- OAC, o qual tem como função principal receber, planejar e enviar os planos de desenvolvimento de cada um dos pedidos. Algumas das principais funções do OAC são: armazenamento dos pedidos conforme a chegada dos mesmos, conhecido como teoria de colas: primeiros pedidos a chegar, primeiros pedidos a sair; definição das zonas, horários, pessoal e equipe de atendimento.

Uma vez executadas as principais atividades pelo OAC, é definida a infraestrutura física e humana para executar o pedido: Os caminhões, com os percorridos e o pessoal definido, entram nas rotas estabelecidas para o atendimento aos cidadãos. O conjunto de atividades planejadas e executadas mencionadas anteriormente integram o atendimento pontual do cliente. Para o controle de atendimento dos pedidos, avalia-se seu estado, julgando se solucionou ou não a solicitação feita pelo cliente: se o serviço foi prestado em sua totalidade, estabelece-se como fim do serviço; se não foi completado, ele entra de novo no Sistema SAC e é armazenado para ser completado em outro plano de roteamento posterior.

O fluxo de transporte dos Resíduos Sólidos Domiciliares inicia-se no depósito dos caminhões, conhecido como Gerência de Remoção Gratuita, localizada na Rua General Polidoro, 68, Botafogo. Ele é o ponto de partida e de chegada dos veículos. Depois, os caminhões dirigem-se até as fontes geradoras de lixo, sendo eles os clientes da zona sul da 
cidade, denominada área de planejamento dois. Uma vez coletados, são transportados até a Estação de Transferência final, ETR-Caju, localizada na Rua Carlos Seidl 1388, Caju, como é apresentado na Figura 3.1:

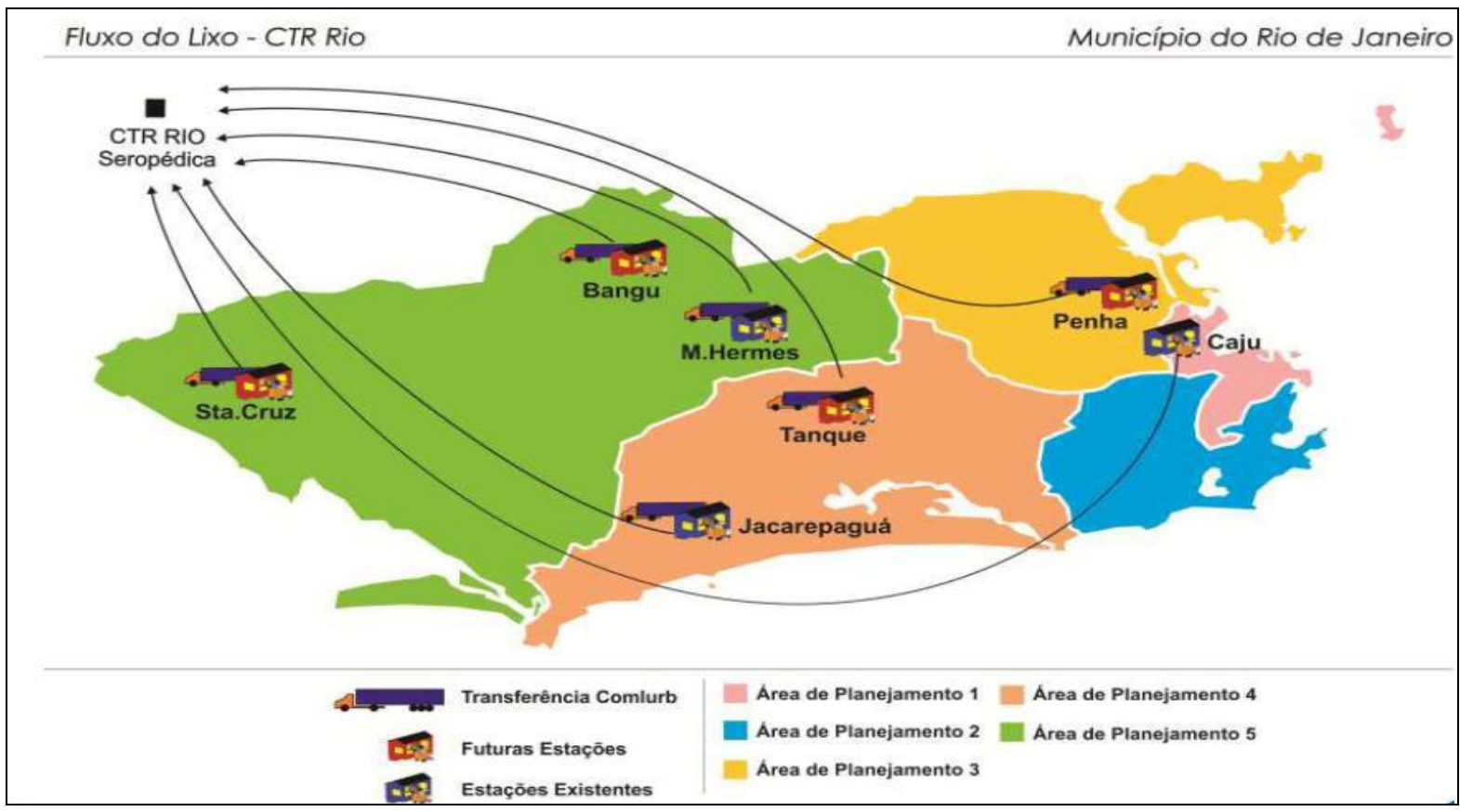

Figura 3.1. Fluxo do Lixo do Centro de Tratamento de Resíduos-CTR Rio. Fonte: [11]

De acordo com a Figura 3.1, na cidade do Rio de Janeiro existem três Estações de transferências principais: Estação de Transferência de Caju, Marechal Hermes e Jacarepaguá. Com o planejamento de crescimento do Projeto Ciclus, a partir do ano 2012 projetou-se a ampliação na cobertura de coleta de Resíduos Sólidos, com a criação de mais quatro estações de transferência: Santa Cruz, Bangu, Penha e Tanque.

Uma vez transportados até a estação de transferência, são planejadas as viagens até o Aterro Sanitário de Seropédica, sendo o ponto final para o esvaziamento e controle.

Para o transporte dos Resíduos Sólidos, existe uma série de veículos com características próprias para a execução da atividade. Segundo [1], existem dois tipos de caminhões que trabalham no PRG: o primeiro grupo é composto por três caminhões basculantes de 7 metros cúbicos, com capacidade de carga máxima de 7000 quilogramas; e o segundo grupo é composto por um caminhão basculante de 3 metros cúbicos, com capacidade de carga máxima de 3000 quilogramas.

O horário de atendimento ao usuário no Serviço de Remoção Gratuita é das 7:00 horas as 22:40 horas de segunda-feira a sábado. Os horários de retirada dos materiais não são agendados na Gerência Zona Sul, ou seja, os caminhões podem passar em qualquer momento do dia estabelecido para fazer a coleta de Resíduos Sólidos Domiciliares. [1] argumenta que, somente para as seguintes exceções pode-se programar a retirada dos rejeitos com a opção de agendamento incluindo o telefone fixo do cliente: caso não tenha local para acondicionar o material ou caso não tenha responsável para receber o funcionário da coleta no local.

Uma vez gerado o pedido, a Gerência de Remoção Gratuita tem um prazo de atendimento de até 10 dias. Se for necessário um novo pedido, ele deverá ser feito após 10 dias da data de atendimento do pedido anterior. Para o caso dos pedidos não atendidos ou cancelados, eles poderão ser reprogramados imediatamente. 


\section{SISTEMAS DE INFORMAÇÕES GEOGRÁFICAS PARA TRANSPORTE (SIG-T)}

De acordo com [16], os Sistemas de Informações Geográficas (SIG) podem ser definidos como o "conjunto de hardware, software, dados geográficos e alfanuméricos, projetados para eficientemente, capturar, armazenar, atualizar, manipular, analisar e apresentar informações referenciadas geograficamente".

Para o uso na área de transportes, foram desenvolvidos especificamente Sistemas de Informações Geográficas responsáveis pelo armazenamento, amostragem, gerenciamento e análise dos dados de transporte. Eles são conhecidos como os SIG-T e podem ser definidos como uma integração do SIG e um Sistema de Informação de Transporte (SIT). [20]

O SIG-T mais reconhecido é o software TransCAD. [9] descreve o software como um SIG-T que aplica rotinas específicas para o desenvolvimento de problemas de pesquisa operacional, logística e transporte em geral. Além disso, o software tem uma estrutura modular aberta que permite solucionar problemas de roteirização e programação de veículos com os procedimentos utilizados pelo próprio usuário.

As aplicações possíveis dentro do software TransCAD permitem a interação entre os elementos de transporte e as infraestruturas espaciais. De acordo com [6], o SIG-T permite simular cenários para as análises de redes (rotas mais curtas, mais rápidas, menor custo), planejamento de transportes e demandas de viagens (geração/distribuição de viagens, modelagens de escolha modal e alocação da demanda), roteirização e logística (operação de coleta de entrega, planejamento da distribuição, manutenção de instalações, coleta de Resíduos Sólidos, percorridos de leituras como energia, etc.), regionalização e alocação de instalações (localização de depósitos, delimitação de redes de serviços, etc.).

\subsection{LIMITAÇÕES DO USO DO SIG-T TRANSCAD}

Embora os resultados das rotinas praticadas dentro do software TransCAD permitam uma tomada de decisão baseada em critérios espaciais com a análise das informações, existem limitações para os problemas de roteirização simulados pelo software. As principais desvantagens que apresentam a simulação de dados dentro do TransCAD são as seguintes [17]: mistura de produto, ocorre quando existem diferentes produtos a serem entregues com o mesmo veículo, mas alguns veículos possuem restrição de tipo de produto que podem carregar; início-término da rota, acontece para veículos que não precisam retornar ao depósito no final do roteamento ou aqueles que podem visitar os depósitos durante o roteamento.

Além das restrições do software mencionadas anteriormente, [19] argumenta algumas limitações na simulação dos dados dentro do SIG-T TransCAD: alto consumo de tempo para o cálculo de previsão de tráfego; alguns movimentos ou sentidos das rodovias podem não ser razoáveis em comparação com a realidade, limitando ao pesquisador a tomada de decisões; as rotinas desenvolvidas devem ser avaliadas constantemente na procura de novos resultados e no controle de erros excessivos; se não melhora o processo, o pesquisador tem que ajustar os resultados para que sejam os mais razoáveis; como último, é indispensável um treinamento ou conhecimentos básicos para o uso do software.

\section{PROBLEMA DE ROTEIRIZAÇÃO DE VEÍCULOS COM JANELA DE TEMPO}

[4] descreve o Problema de Roteirização dos Veículos com Janela de Tempo (PRVJT) como uma generalização do Problema de Roteirização de Veículos com restrições adicionais denominadas Janelas de Tempo, onde serão satisfeitas as necessidades dos clientes, considerando termos de horário e realização do serviço, como é apresentado na Figura 5.1. Para cada cliente existe uma janela de tempo, com o intervalo [t1,t2], sendo o t1 o tempo de início da janela, e o t2 o tempo do fim. Só neste intervalo de tempo o cliente deverá ter o seu atendimento. Considera-se também janela de tempo para o depósito onde, de acordo com o horário de funcionamento, os veículos devem sair e retornar a ele. 


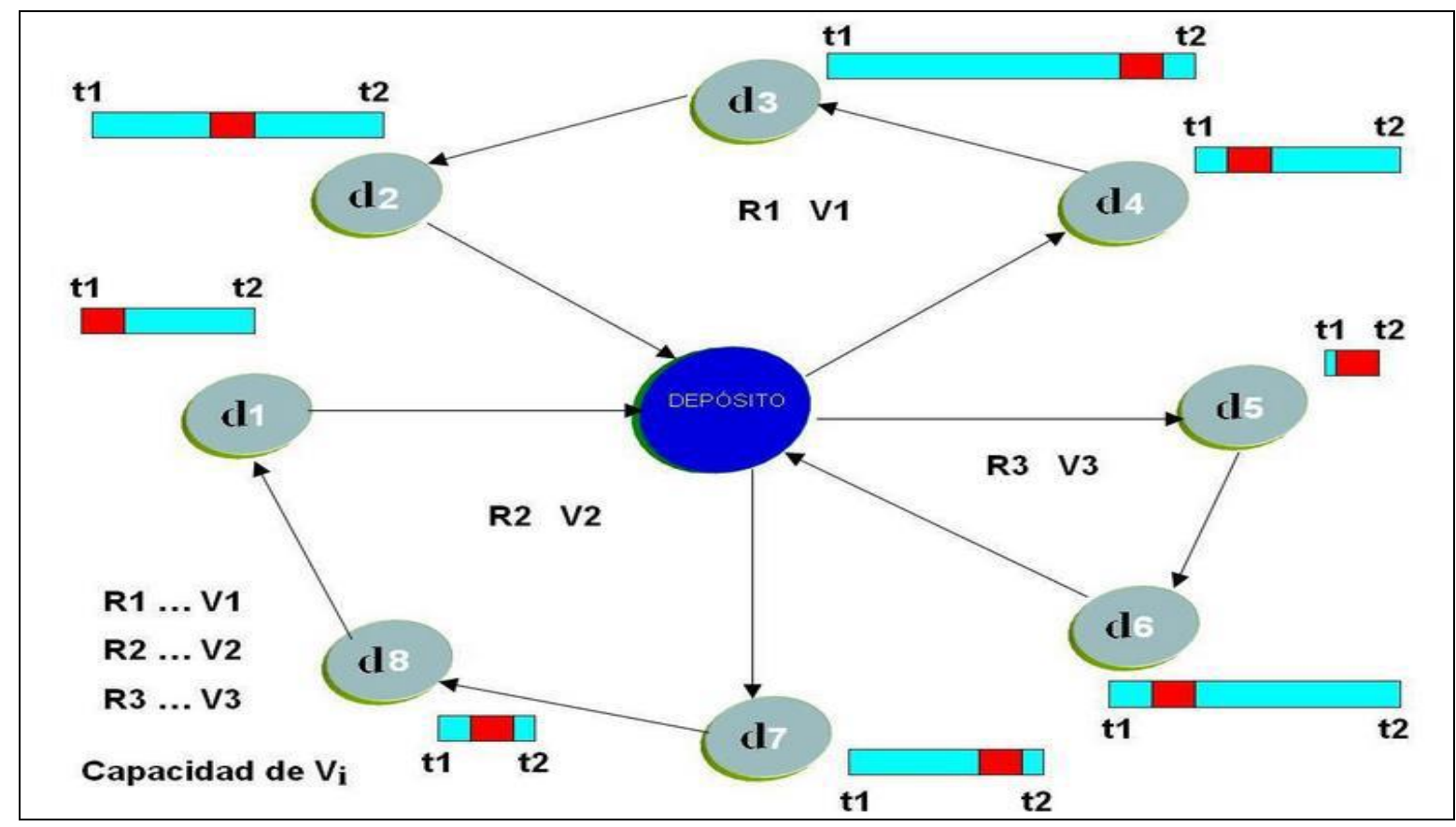

Figura 5.1. Exemplo do Problema de Roteirização de Veículos com Janelas de Tempo Fonte: [7]

Na figura 5.1, tem-se um depósito principal (Depósito), o qual é o ponto de início e fim do conjunto de rotas R (R1, R2 e R3) para o conjunto de Veículos V (V1, V2 e V3), os quais estão sujeitos a uma capacidade (Capacidade de Vi). O objetivo da criação dos roteiros é o atendimento do conjunto de clientes com demandas $\mathrm{d}(\mathrm{d} 1, \mathrm{~d} 2, \ldots \mathrm{d} 8)$, onde cada um deles tem uma janela de tempo de início (t1) e de fim (t2) na qual devem ser servidos.

O presente problema de roteirização existe como rotina no SIG-T TransCAD versão 5.0 para resolver o caso de estudo deste trabalho. O material acadêmico descrito pelo autor do software [5] não especifica o algoritmo utilizado, mas faz referência à pesquisa desenvolvida por [18] como suporte matemático. Revisões com respeito aos PRVJT podem ser encontradas nos trabalhos de [13], [8] entre outros.

\section{ESTUDO DE CASO}

A cidade do Rio de Janeiro está localizada na região sudoeste do estado do Rio de Janeiro. Segundo [12], a cidade possui uma população de 6.453 .682 habitantes.

De acordo com [21], atualmente na cidade geram-se 53\% de Resíduos Sólidos úmidos (matéria orgânica) e 47\% de Resíduos Sólidos secos (matéria inorgânica). A fração inorgânica produzida pelo estado do Rio de Janeiro está composta por material plástico (19\%), papel (16\%), vidro (3\%) e metais (2\%).

Os materiais provenientes da categoria "outros resíduos" (7\%) são conhecidos como rejeitos denominados "resíduos especiais". Estes rejeitos fazem parte do grupo de resíduos coletados pelo PRG e são parte do grupo de resíduos de estudo da presente pesquisa.

Atualmente, existem duas Gerências principais para a coleta de Resíduos Sólidos da cidade: a primeira é a Gerência de Resíduos Sólidos Orgânicos e a segunda é a Gerência de Resíduos Sólidos Inorgânicos, também conhecida como Gerência de Coleta Seletiva, responsável pela gestão dos recicláveis (vidro, metal, plástico, papel, etc.). O PRG pertence à última mencionada, sendo considerado como um subprograma especial de coleta de Resíduos Sólidos Domiciliares da cidade.

Para o presente estudo, foi escolhida como área de pesquisa a zona sul da cidade do Rio de Janeiro. O PRG abrange os seguintes bairros: Jardim Botânico, Lagoa, Ipanema, Gávea, Leblon, Botafogo, Copacabana, Flamengo, Leme, Vidigal, Urca, Catete, Glória, São 
Conrado, Cosme Velho, Laranjeiras, Humaitá, entre outros.

Dentro do programa, cada ligação ao serviço 1746 gera um pedido de coleta de Resíduos Sólidos Domiciliares, representado por um ponto no presente estudo. A gerência faz atendimento de pedidos com frequência diária, ou seja, todos os pedidos dos clientes são armazenados na base de dados e enviados ao gerente ao final do dia, com o objetivo de fazer o planejamento para o dia seguinte. Aqueles que não forem cumpridos ou não forem planejados ficam na espera, seguindo o comportamento de chegada e os parâmetros de atendimento.

No atendimento dos serviços solicitados pelos clientes, o PRG é desenvolvido considerando uma série de restrições de tempo, denominadas Janelas de Tempo sendo apresentadas na figura 6.1:

\begin{tabular}{|c|c|c|c|c|c|c|c|}
\hline \multicolumn{4}{|c|}{ PRIMEIRO TURNO } & \multicolumn{4}{c|}{ SEGUNDO TURNO } \\
\hline 7:00 14:20 14:40 & $\begin{array}{c}\text { Retorno } \\
\text { ao } \\
\text { Depósito }\end{array}$ & $\begin{array}{c}\text { Entrega de } \\
\text { turno }\end{array}$ & $\begin{array}{c}\text { Atendimento de } \\
\text { Clientes }\end{array}$ & $\begin{array}{c}\text { Esvaziamento } \\
\text { Atendimento de } \\
\text { Clientes }\end{array}$ & $\begin{array}{c}\text { Esvaziamentorno } \\
\text { ao } \\
\text { Depósito }\end{array}$ & $\begin{array}{c}\text { Entrega de } \\
\text { turno }\end{array}$ \\
\hline
\end{tabular}

Figura 6.1. Distribuição das Janelas de Tempo do Programa de Remoção Gratuita

Fonte: autor

A COMLURB terceiriza o serviço de transporte com a empresa LOCALIX, empresa local de serviços logísticos. A equipe disponível para o atendimento dos pedidos está composta por quatro caminhões fixos, os quais podem ser substituídos em caso de quebra ou manutenção por duas frotas alternativas (extras) com a mesma capacidade.

Com respeito ao destino final dos rejeitos, o PRG coleta, transporta e esvazia os resíduos sólidos na estação de transferência, ponto de disposição final dos rejeitos na presente pesquisa. Desde a estação de Caju, são planejadas diferentes viagens até o Aterro Sanitário de Seropédica, o qual é controlado por uma gerência alternativa.

Identificou-se que os roteiros feitos pelos caminhões não seguem regras ou estudos técnicos de roteirização. Os percorridos são definidos baseados na experiência dos motoristas e o pessoal administrativo da Gerência de Remoção Gratuita, gerando uma diminuição da produtividade e qualidade do serviço prestado à sociedade.

Com o objetivo de apoiar o sistema de planejamento dos roteiros de coleta de Resíduos Sólidos Domiciliares do PRG, é utilizado o SIG-T TransCAD versão 5.0 para o planejamento, execução, análise e controle dos itinerários.

\section{RESULTADOS E ANÁLISE}

Os resultados obtidos para o planejamento das rotas dos caminhões foram produzidos por meio do software TransCAD versão 5.0. A rotina utilizada para a criação dos roteiros foi o Vehicle Routing Problem, também conhecido como o Problema de Roteirização de Veículos, considerando as restrições de tempo, denominadas janelas de tempo.

A criação dos itinerários e os roteiros para os caminhões inclui a geração do plano para oito veículos por dia: H21, H22, H38 e H46 no caso do primeiro turno; H42, H44, H76 e H92 para o caso do segundo turno. É importante esclarecer que a frota disponível para o serviço de coleta dos rejeitos é somente quatro veículos e os outros quatro foram adicionados como apoio na simulação do software para a definição do segundo turno de serviço. Com isso, a relação dos veículos no problema analisado é a seguinte: $\mathrm{H} 21=\mathrm{H} 42 ; \mathrm{H} 22=\mathrm{H} 44 ; \mathrm{H} 38=\mathrm{H} 76$; H46=H92. As análises feitas consideram uma amostra de cinco dias de coleta de Resíduos Sólidos Domiciliares, sendo os dias 01, 02, 03, 04 e 07 de outubro de 2014.

O planejamento das rotas dos veículos considera as informações fornecidas pela Gerência de Remoção Gratuita (cadastros de itinerários de coleta). Utilizaram-se também indicadores adicionais, como o exemplo apresentado na Tabela 7.1. 
TABELA 7.1 - Indicadores das Rotas do PRG para o veículo H21 em 01/10/2014.

\begin{tabular}{|l|c|c|c|c|c|c|c|}
\hline Indicador & Un. & Turno & $\begin{array}{c}\text { Depósito/ } \\
\text { Setor }\end{array}$ & Setor & $\begin{array}{c}\text { Setor/ } \\
\text { Esvaziado }\end{array}$ & $\begin{array}{c}\text { Esvaziado } \\
\text { / Depósito }\end{array}$ & Total \\
\hline Percurso & $\mathrm{Km}$ & & 3 & 11 & 7 & 16 & 37 \\
Tempo & $\mathrm{h}$ & 1 & $0: 20$ & $4: 52$ & $1: 10$ & $0: 37$ & $6: 59$ \\
Clientes & $\mathrm{Un}$. & & 0 & 19 & 0 & 0 & 19 \\
Kg Colet. & $\mathrm{Kg}$ & & 0 & 4302 & 0 & 0 & 4302 \\
\hline
\end{tabular}

Fonte: [1], [6]

Com os dados de entrada, o seguinte passo foi configurar o software e processar as informações dentro do TransCAD versão 5.0 na criação das rotas para os veículos, considerando os tempos e as distâncias entre cada par origem-destino. Uma vez feito, obtémse as saídas com o itinerário detalhado das rotas individualizadas para cada veículo. $\mathrm{O}$ exemplo do itinerário para o caminhão H21 é apresentado na Figura 7.1.

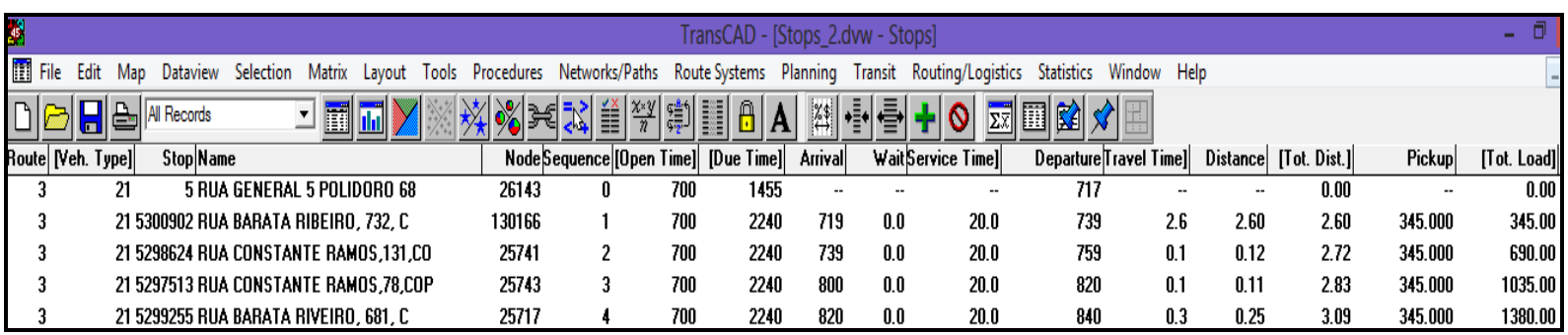

Figura 7.1. Exemplo do itinerário para o veículo H21 do SIG-T, 01/10/ 2014.

Fonte: autor

Uma vez obtidas as informações, inicia-se o processo de análise dos resultados, apresentados na Tabela 7.2:

TABELA 7.2 - Análise para o Roteiro do veículo H21 em 01/10/2014.

\begin{tabular}{|c|c|c|c|c|c|c|c|c|}
\hline Dia & Turno & Veículo & (Km_1) & (Km_2) & (\%_1) & (h_1) & (h_2) & (\%_2) \\
\hline 01 out & 1 & $\mathrm{H} 21$ & 37 & 34,3 & 7,30 & $6: 59$ & $7: 34$ & $-8,35$ \\
\hline
\end{tabular}

Fonte: adaptado de [14].

Onde:

(Km_1): Distância total percorrida no itinerário da COMLURB

(Km_2): Distância total percorrida com o uso do SIG-T.

(\%_1): Redução percentual da distância total percorrida

(h_1): Tempo de Viagem total no Itinerário da COMLURB

(h_2): Tempo de Viagem total com o uso do SIG-T

(\%_2): Redução percentual do tempo de viagem total

De acordo com os resultados anteriores, a distância total percorrida pelo veículo com o uso do SIG-T TransCAD versão 5.0 é reduzida em 7,30\% em comparação com a distância total feita no itinerário da COMLURB. Porém, o tempo utilizado pelo SIG-T aumentou 8,35\% em comparação com o plano real. $\mathrm{O}$ anterior deve-se, principalmente, ao fato de que o software TransCAD conseguiu fazer um itinerário com um número de clientes a serem atendidos maior (20 em total) em comparação com o plano feito pela COMLURB (19 em total). Além disso, no plano de roteirização criado pelo SIG-T, o tempo de serviço é considerado fixo em todos os clientes, fato que não acontece no plano real onde, se o serviço é 
cumprido num intervalo de tempo menor, o veículo dirige-se automaticamente ao seguinte cliente.

Para localizar geograficamente os pontos de serviço a serem atendidos, o SIG-T TransCAD cria a camada de pontos chamada de paradas (stops) que contém a informação espacial dos dados para o itinerário a ser executado pelo veículo. O anterior é conhecido como o mapa de roteiro para o veículo; para o caso do caminhão H21 é apresentado na Figura 7.2:

Conhecendo as informações espaciais e o itinerário a ser percorrido pelo caminhão $\mathrm{H} 21$, a responsabilidade agora deve ser assumida pelo recurso humano, encargado de planejar, executar, analisar e controlar os roteiros estabelecidos para o conjunto de caminhões do programa nos dias de serviço, de segunda-feira a sábado.

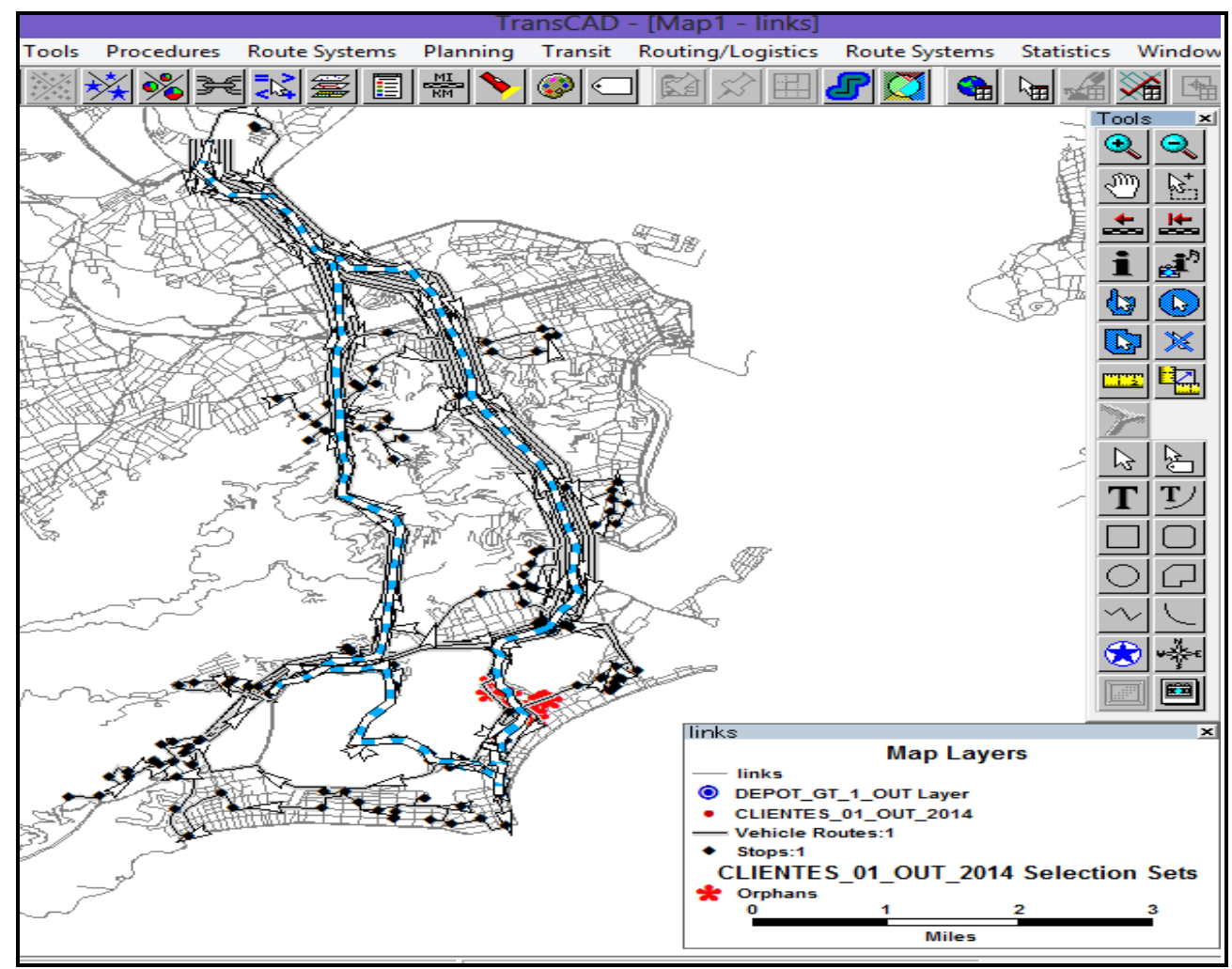

Figura 7.2. Mapa de Roteiro para o veículo H21 do SIG-T, 01/10/ 2014.

Fonte: autor

Existem pontos que ficam fora dos roteiros a serem servidos pelos veículos. Eles são conhecidos como os pontos órfãos (Orphans). Para atender o conjunto de clientes órfãos, duas opções são possíveis: a primeira é criar uma rota para o conjunto de pontos órfãos e atendê-la num dia posterior; a segunda opção é deixar esses pontos como dados de entrada para o dia seguinte, processá-los com o conjunto de clientes a servir e tentar inclui-los.

De uma forma geral, os resultados obtidos evidenciaram que existe uma diminuição nos percorridos para os caminhões com o uso do SIG-T. Para os tempos, existe um incremento a ser estudado e controlado, devido ao fato de que as informações de entradas para a variável mencionada estão sujeitas a procedimentos acadêmicos que, de certa forma, podem diminuir a objetividade da pesquisa e gerar improdutividade dentro do PRG.

\section{CONCLUSÕES}

O SIG-T é uma ferramenta fundamental para o planejamento, execução, análise e controle das rotas de coleta dos Resíduos Sólidos Domiciliares. A implementação do software TransCAD versão 5.0 evidencia que existem métodos matemáticos, como o problema de 
roteirização desenvolvido na presente pesquisa, que permitem resolver quantitativamente o procedimento de criação dos itinerários de coleta.

Um fator importante a ser considerado no sucesso da pesquisa é a aceitação do SIG$\mathrm{T}$ por parte dos recursos humanos da empresa. Com tal apoio, é possível desenvolver a metodologia eficazmente dentro do Programa de Remoção Gratuita.

Do ponto de vista quantitativo, evidenciaram-se melhoras para as distâncias percorridas pelos caminhões dentro do programa. Porém, existem fatores de improdutividade para os tempos estabelecidos. É importante que a implementação do SIG-T considere as restrições de tempo reais para adaptar-se ao regulamento de coleta estabelecido pela empresa municipal.

O planejamento das rotas com o apoio do SIG-T é concebido como um método para o melhoramento contínuo do processo. As informações geradas pelo software permitem aperfeiçoar o serviço ao cliente e obter uma base de dados de referência para tomada de decisões e futuros estudos.

Como conclusão geral, a implementação da metodologia permite identificar os principais indicadores, obstáculos e restrições para o procedimento de planejamento de rotas com o uso do SIG-T, o qual incrementa a possibilidade de obtenção de uma análise objetiva para os dados. Espera-se que o presente estudo seja uma contribuição acadêmica para esse problema específico de roteirização e para o desenvolvimento de futuras pesquisas nessa área.

\section{REFERÊNCIAS BIBLIOGRÁFICAS}

[1] ARAÚJO, F.(2014). Diretoria de Serviços Especiais e Ambientais (DSA).Gerência de programação e controle (AGC).Companhia Municipal de Limpeza Urbana (COMLURB). Notas do programa de atendimento de remoção gratuita. Gerência ADS Zona Sul.

[2] BARBOSA, L.(2004). Gerenciamento de resíduos sólidos urbanos no norte de Minas Gerais: Estudo relativo à implantação de unidades de reciclagem e compostagem a partir de 1997. Universidade Federal de Minas Gerais. Programa de Pós-graduação em saneamento, meio ambiente e recursos hídricos. Vol.1. P 23-63.

[3] BASTOS, M.(2009). Plano Municipal de Gestão Integrada de Resíduos Sólidos. Disponível na web: http://www.rio.rj.gov.br/web/smac/residuos-solidos. Acesso em: 12 de Agosto de 2014.

[4] BATISTUS, D. (2009). O Problema de Roteirização de Veículos com restrições de Janelas de Tempo: Uma proposta de Solução por meio da Metaheurística Scatter Search. Dissertação de Doutorado. Programa de Pós-graduação em Engenharia de Produção. Universidade Federal de Santa Catarina. Vol.1. P.26-126.

[5] CALIPER CORPORATION (1990). Reference Manual 2.0. Neewton, Masachusets.

[6] CASTRO, L.(2006). Avaliação do serviço de coleta de resíduos sólidos domiciliares em cidade de médio porte utilizando sistemas de informações geográficas e receptores do sistema de posicionamento global. Universidade Federal de Uberlândia. Vol.1. P 10-18.

[7] CRUZ, M.(2014).El Problema del Ruteo Vehicular con Ventanas de Tiempo. Centro de investigación en Ingeniería y Ciencias Aplicadas-CIICAP. Universidad Autónoma del Estado de Morelos,México. Disponível na Web: http:/ /www. gridmorelos. uaem.mx/ $\sim$ mcruz//doctor/vrptw.htm. Acesso em: 14 de Janeiro de 2015.

[8] DA SILVA JUNIOR ,O. (2009). Comparação de modelos exatos para solução do Problema de Roteirização de Veículos com Janela de Tempo. XLII SBPO. Departamento de Engenharia de Produção. Pontifícia Universidade do Rio de Janeiro. Vol.1.P.1-8. 
[9] DE OLIVEIRA, R.(2011). Logística Reversa: A utilização de um Sistema de Informações Geográficas na Coleta Seletiva de Materiais Recicláveis. Dissertação de Mestrado como requisito para a obtenção de titulo de Mestre em Engenharia de Produção. Universidade Federal de Itajubá. Vol.1. P.8-152.

[10] FILHO, C.(2013). Panorama dos resíduos sólidos no Brasil. Associação Brasileira de Empresas de Limpeza-ABRELPE. Vol. 1. Pag.28-47.

[11] FONTI, A.(2012). COMLURB sustentável. Presidência da Companhia Municipal de Limpeza Urbana-COMLURB. Vol.1. P.1-26.

[12] INSTITUTO BRASILEIRO DE GEOGRAFIA E ESTATISTICA (IBGE). (2014). Estimativa da população residente no Brasil e unidades da federação com data de referencia em $1^{\circ}$ de Julho de 2014. Disponível em web:http://www.ibge.gov.br/home/estatistica/populacao/estimativa2014/estimativa_tcu. shtm. Acesso em: 27 de Dezembro de 2014

[13] LARSEN, J. (1999). Parallelization of the Vehicle Routing Problem with Time Windows. Tese (Ph.D.). Department of Mathematical Modeling. Technical University of Denmark.Vol.1.P. 31-294.

[14] LIMA, R.LIMA,J.SILVA,T.(2012). Roteirização em Arcos com um Sistema de Informações Geográficas para transportes: aplicação em coleta de resíduos sólidos urbanos. Journal of Transport Literature. Universidade Federal de Itajubá (UNIFEI).Vol.6.P.180-196.

[15] MAGALHÃES, D. (2008). Elementos para o diagnóstico e gerenciamento dos resíduos sólidos urbanos do município de Dores de Campos - MG. Universidade Federal de Juiz de Fora. Curso de especialização em análise ambiental. Vol.1. P.19-29.

[16] NETO, A.LIMA, R.(2006). Roteirização de Veículos de uma rede atacadista com o auxilio de Sistemas de Informações Geográficas (SIG). Revista Pesquisa e Desenvolvimento Engenharia de Produção. Universidade Federal de Itajubá. Instituto de Engenharia de Produção e Gestão. Vol.5. P.1-22.

[17] PELIZARO, C.(2000). Avaliação do Desempenho do Algoritmo de um Programa Comercial para Roteirização de Veículos. Dissertação de Mestrado. Escola de Engenharia de São Carlos. Departamento de Engenharia de Transportes. Universidade de São Paulo (USP).Vol.1.P.104-166.

[18] SOLOMON, M. (1987). Algorithms for the Vehicle Routing and Scheduling Problems with Time Windows Constrains.Operations Research.Vol.2.P.254.

[19] STONE, J.SAUR, G.LETCHWORTH, W.(2002). Guidelines for NCDOT Project-Level Traffic Forecasting Procedures. Department of Civil Engineering. North Carolina State University. Vol.1.P. 72-187.

[20] VONDEROHE, A.TRAVIS, L.SMITH, R.TSAI, V. (1993). Adaptation of Geographic Information Systems for Transportation. Transportation Research Board. National Research Council.P.359.

[21] ZVEIBIL, V.SANTANA, A. MOTA, D.SOARES, E. PINHEIRO, J.ARAÚJO, M.SAROLDI, M.(2013).Plano Estadual de Resíduos Sólidos ,do Rio de Janeiro- PERS. Relatório Síntese 2013.Gestão de Resíduos Sólidos do Estado do Rio de Janeiro. Instituto Estadual do Ambiente-INEA.Vol.1.P.1-6 